\title{
Microglial response to increasing amyloid load saturates with aging: a longitudinal dual tracer in vivo $\mu$ PET-study
}

\author{
Tanja Blume ${ }^{1,2 \dagger}$, Carola Focke ${ }^{1 \dagger}$, Finn Peters ${ }^{2 \dagger}$, Maximilian Deussing ${ }^{1}$, Nathalie L. Albert ${ }^{1}$, Simon Lindner ${ }^{1}$, \\ Franz-Josef Gildehaus ${ }^{1}$, Barbara von Ungern-Sternberg ${ }^{1}$, Laurence Ozmen ${ }^{3}$, Karlheinz Baumann ${ }^{3}$, Peter Bartenstein ${ }^{1}$, \\ Axel Rominger ${ }^{1,4,6}$, Jochen Herms ${ }^{2,5,6+}$ and Matthias Brendel ${ }^{1,6^{*}+}$
}

\begin{abstract}
Background: Causal associations between microglia activation and $\beta$-amyloid (A $\beta$ ) accumulation during the progression of Alzheimer's disease (AD) remain a matter of controversy. Therefore, we used longitudinal dual tracer in vivo small animal positron emission tomography ( $\mu$ PET) imaging to resolve the progression of the association between $A \beta$ deposition and microglial responses during aging of an $A \beta$ mouse model.

Methods: APP-SL70 mice ( $N=17$; baseline age 3.2-8.5 months) and age-matched C57BI/6 controls (wildtype $(\mathrm{wt})$ ) were investigated longitudinally for 6 months using $\mathrm{A} \beta$ (18F-florbetaben) and $18 \mathrm{kDa}$ translocator protein (TSPO) MPET (18F-GE180). Changes in cortical binding were transformed to Z-scores relative to wt mice, and microglial activation relative to amyloidosis was defined as the Z-score difference (TSPO-A $\beta$ ). Using 3D immunohistochemistry for activated microglia (Iba-1) and histology for fibrillary A $\beta$ (methoxy-X04), we measure microglial brain fraction relative to plaque size and the distance from plaque margins.

Results: A -PET binding increased exponentially as a function of age in APP-SL70 mice, whereas TSPO binding had an inverse U-shape growth function. Longitudinal Z-score differences declined with aging, suggesting that microglial response declined relative to increasing amyloidosis in aging APP-SL70 mice. Microglial brain volume fraction was inversely related to adjacent plaque size, while the proximity to $A \beta$ plaques increased with age.
\end{abstract}

Conclusions: Microglial activity decreases relative to ongoing amyloidosis with aging in APP-SL70 mice. The plaqueassociated microglial brain fraction saturated and correlated negatively with increasing plaque size with aging.

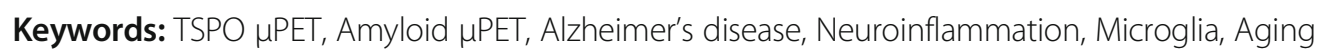

\section{Background}

The progressive accumulation of senile plaques composed of $\beta$-amyloid $(A \beta)$ is a main pathological hallmark of Alzheimer's disease (AD), the most common dementing disorder in the elderly. The $A \beta$ accumulation promotes synaptic loss and neuronal degeneration apparently by activating microglia, the resident macrophages of the brain [1-4]. In the healthy brain, microglia cells are long-lived

\footnotetext{
* Correspondence: matthias.brendel@med.uni-muenchen.de

†Tanja Blume, Carola Focke, Finn Peters, Jochen Herms and Matthias Brendel contributed equally to this work.

${ }^{1}$ Department of Nuclear Medicine, University Hospital, LMU Munich, Marchioninistraße 15, 81377 Munich, Germany

${ }^{6}$ Munich Cluster for Systems Neurology (SyNergy), Munich, Germany Full list of author information is available at the end of the article
}

cells using highly motile processes to survey parenchymal territory for the presence of pathogens and cell debris. In addition, microglia secrete factors that support neuronal survival and synaptogenesis [5]. In the early stages of AD, microglia migrate towards amyloid deposits and express certain cell-surface receptors to promote the clearance and phagocytosis of $A \beta$ [6-8]. Furthermore, deficits in microglia activation favor accelerated amyloid deposition [9]. However, it has been hypothesized that microglial reactions are overwhelmed by the massive $A \beta$ deposition in later $\mathrm{AD}$ stages $[10,11]$. This suggestion is supported by the finding that plaque-associated microglia ultimately show decreased expression of $A \beta$-binding receptors, which leads to a significant reduction in $A \beta$ degradation

(c) The Author(s). 2018 Open Access This article is distributed under the terms of the Creative Commons Attribution 4.0 International License (http://creativecommons.org/licenses/by/4.0/), which permits unrestricted use, distribution, and 
by microglia in the aging brain [11]. Moreover, plaqueassociated microglial cells show a threefold higher mortality rate compared to non-plaque-associated microglia in vivo [12].

The use of small animal positron emission tomography ( $\mu \mathrm{PET}$ ) with $\mathrm{A} \beta$ tracers enables longitudinal investigations of cerebral amyloidosis in rodents in vivo $[13,14]$. Confirmation of the hypothesis of a ceiling effect in microglial reactions has been hampered by the technical difficulty in following the fate of aging microglial cells in living mice. The past decade has seen the introduction of $\mathrm{A} \beta-\mu \mathrm{PET}$ in rodents $[15,16]$, using the same radioligands employed in the clinical routine for the differential diagnosis of $\mathrm{AD}[17,18]$. A series of PET radiotracers targeting the microglial marker $18-\mathrm{kDa}$ translocator protein (TSPO), formerly known as the peripheral benzodiazepine receptor (PBR) [19-23], has been developed in recent years.

The basal availability of TSPO binding sites is low in the healthy living brain (21), such that local upregulation presents a sensitive marker for the detection of microglial activation in afflicted brain regions [24-26]. This is supported by findings of elevated TSPO expression in the hippocampus and the frontal, temporal, and parietal cortices of postmortem AD brain [25, 27, 28]. Our group has recently established cross-sectional dual tracer $\mu$ PET imaging of $A \beta$ and TSPO in transgenic AD mouse models [29]. Given this background, we aimed in the present longitudinal A $\beta / T S P O$ double tracer $\mu \mathrm{PET}$ study to explore the longitudinal association between amyloidosis and microglial response during aging of an amyloid mouse model in vivo. By using mice with a range of baseline age, we were able to perform correlation analysis with the longitudinal biomarker progression over 7 months. Final immunohistochemistry supported the interpretation of $\mu$ PET results by mapping of individual plaques and microglial cells.

\section{Methods}

\section{Animals and study design}

All experiments were carried out in compliance with the National Guidelines for Animal Protection, Germany, and with the approval of the regional animal care committee (Regierung Oberbayern) and were overseen by a veterinarian. Animals were housed in a temperature- and humiditycontrolled environment with a $12 \mathrm{~h}$ light-dark cycle, with free access to food (Sniff, Soest, Germany) and water.

All experiments were performed in APP-SL70 mice, a mouse-line produced by Roche (Basel, Switzerland), $(N=17$, baseline age: 3.2 to 8.5 months of age: 3.2 to 5.0 months $(N=4) ; 5.1$ to 6.8 months $(N=6)$; and 6.9 to 8.5 months $(N=7))$. First fibrillar $\mathrm{A} \beta$ deposits in this mouse-line appear as early as 2.5 months of age, similar to the mouse line used by Blanchard [30].
Congophilic plaques are observed starting from 5 to 6 months of age. Protein levels of $A \beta 40$ and $A \beta 42$ start to increase from 3 months of age and range around $1 \mathrm{ng} / \mathrm{mg}$ brain at 6 months, $25 \mathrm{ng} / \mathrm{mg}$ brain at 9 months, and $90 \mathrm{ng} / \mathrm{mg}$ brain at 12 months of age. $\mu$ PET examinations (A $\beta$ and TSPO) were performed in a longitudinal design at baseline (0 months), follow-up $(+2.2$ months $/ 11$ weeks $)$ and terminal age (+ 6.3 months/29 weeks). Serial $\mu$ PET scans of both tracers deriving from a total of 30 age-matched C57Bl6 mice (wt) served as control data. All mice were killed after terminal scanning, followed by rapid brain removal and performance of immunohistochemistry analyses. The study design is illustrated in Fig. 1.

\section{Radiochemistry}

Radiosynthesis of $\left[{ }^{18} \mathrm{~F}\right]$-GE180 was performed as previously described [23], with slight modifications [29]. This procedure yielded product with radiochemical purity exceeding $98 \%$, and molar activity of $1400 \pm 500$ gigabecquerel $(\mathrm{GBq}) / \mu \mathrm{mol}$ at end of synthesis. Radiosynthesis of $\left[{ }^{18} \mathrm{~F}\right]$-florbetaben was performed as described earlier $[16,31]$.

$\mu$ PET data acquisition, reconstruction and preprocessing All $\mu$ PET procedures followed an established standardized protocol for radiochemistry, acquisition, and post-processing $[29,32]$. Mice were anesthetized with isoflurane $(1.5 \%$, delivered at $3.5 \mathrm{l} / \mathrm{min}$ ) and placed in the aperture of the Siemens Inveon DPET (Siemens, Knoxville, USA), as described previously [33]. In brief, we made TSPO- $\mu$ PET emission recordings during 60-90 min (p.i.) per image of $\left[{ }^{18} \mathrm{~F}\right]$-GE180 $\quad(11.2 \pm 1.5$ megabecquerel (MBq)) beta-amyloid- $\mu$ PET emission recordings during 30$60 \mathrm{~min}$ p.i. of $\left[{ }^{18} \mathrm{~F}\right]$-florbetaben $(10.8 \pm 1.5 \mathrm{MBq})$. All images were spatially normalized using automatic algorithms and tracer specific templates [32].

\section{$\mu \mathrm{PET}$ data analyses}

All $\mu$ PET analyses were performed with PMOD (V3.5, PMOD technologies, Basel, Switzerland). Normalization of emission images to standardized uptake value ratio (SUVR) images was performed using a previously validated white matter reference region [29, 32]. A target volume of interest (VOI) was placed in the bilateral frontal cortex $\left(46 \mathrm{~mm}^{3}\right)$ and SUVR $\mathrm{CTX} / \mathrm{WM}$ values were extracted for each individual APP-SL70 and wt mouse at the serial imaging time points for both tracers. Differences of TSPO- and A $\beta-\mu$ PET SUVR between terminal and baseline time points were calculated as percentage change $(\Delta \%) . Z$-scores of the TSPO- and $\mathrm{A} \beta-\mu \mathrm{PET}$ tracer uptake in individual APP-SL70 mice were calculated by normalization to age-matched wt mice. To this end, the mean uptake in age-matched wt 


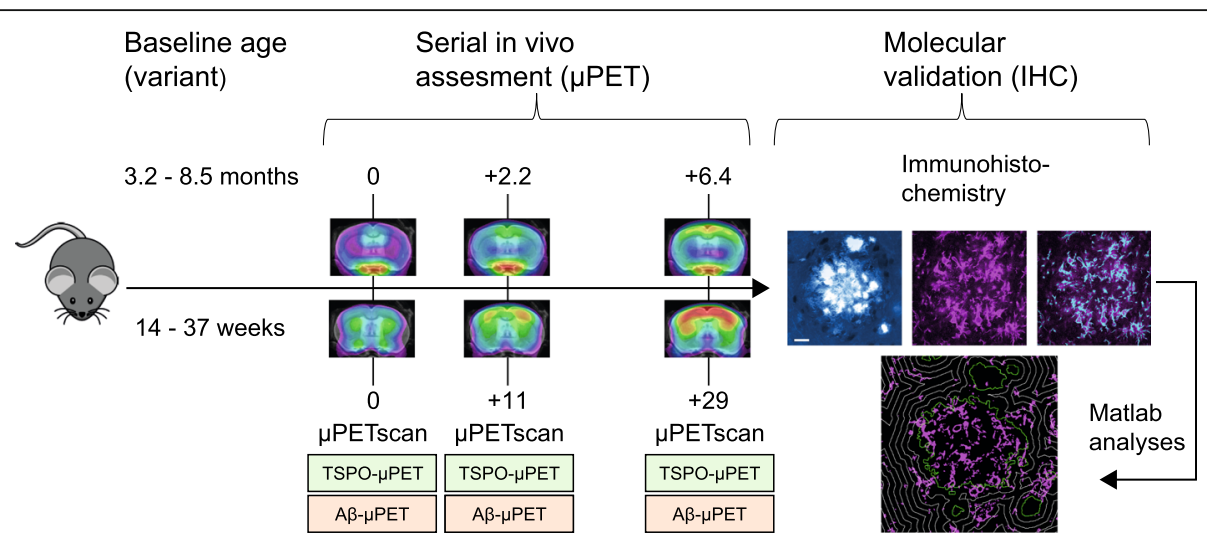

Fig. 1 Schematic illustration of the study design. In vivo $\beta$-amyloid small animal positron emission tomography (Aß- $\mu \mathrm{PET}$ ) and $18 \mathrm{kDa}$ translocator protein (TSPO)- $\mu$ PET imaging was performed in a longitudinal design with baseline examination at 0 months, follow-up at +2.2 months, and terminal examination at +6.3 months in the Alzheimer's disease (AD) mouse model, APP-SL70. Molecular validation of $\mu$ PET results was performed via immunohistochemistry in mid- (11.4 to 12.7 months) and late-aged (13.6 to 15.3 months) APP-SL70 mice after the final $\mu$ PET-scan. Custom-written Matlab software was used to quantify histological results

mice was subtracted from the individual value in APP-SL70 mice (APP-SL70 INDIVIDUAL - wt $\mathrm{MEAN}_{\text {) }}$ ) and the resulting difference divided by the corresponding standard deviation for wt mice to generate an individual $Z$-score value.

$$
Z \text {-score }=\frac{\text { APPSL70 }(\text { INDIVIDUAL })-w t(\text { MEAN })}{w t(S D)}
$$

To establish a readout of microglial activity relative to fibrillary amyloidosis we calculated $Z$-score differences between TSPO- and A $\beta$ - $\mu$ PET. $Z$-scores deriving from the same imaging time point of individual mice (gap $0.35 \pm 1.62$ weeks).

$$
\begin{aligned}
Z \text {-score difference }= & Z \text {-score }(T S P O-\mu \mathrm{PET}) \\
& -Z \text {-score }(A \beta-\mu \mathrm{PET})
\end{aligned}
$$

Immunohistochemistry: acquisition and image analysis Brains intended for immunohistochemistry were fixed by immersion in $4 \%$ paraformaldehyde at $4{ }^{\circ} \mathrm{C}$ for $15 \mathrm{~h}$. Two representative $50-\mu \mathrm{m}$-thick slices per animal were then cut in the axial plane using a vibratome (VT 1000 S, Leica, Wetzlar, Germany). Free-floating sections were permeabilized with $2 \%$ Triton X-100 overnight and blocked with I-Block ${ }^{\mathrm{TM}}$ Protein-Based Blocking Reagent (Thermo Fischer Scientific, Waltham, USA). We obtained immunofluorescence labelling of microglia using an Iba-1 primary antibody (Wako, Richmond, USA) with a dilution of 1:200 in I-Block $^{\mathrm{TM}}$ and the A-21244 secondary antibody (Invitrogen, Carlsbad, USA) with a dilution of 1:500 in I-Block $^{\mathrm{Tm}}$. For histological staining against fibrillar $\mathrm{A} \beta$, we used methoxy-X04 (TOCRIS, Bristol, United Kingdom) with a dilution of $0.01 \mathrm{mg} / \mathrm{ml}$. The unbound dye was removed in three washing steps with PBS, and the slices were then mounted on microscope slides with fluorescent mounting medium (Dako, Santa Clara, USA). Images were acquired with a LSM 780 confocal microscope (Zeiss, Oberkochen, Germany) equipped with a $40 x / 1.4$ oil immersion objective. The excitation wavelength for Iba-1 detection was $633 \mathrm{~nm}$ and emission was detected from 638 to $755 \mathrm{~nm}$. For methoxy-X04, the excitation wavelength was $405 \mathrm{~nm}$ and emission was detected from 403 to $585 \mathrm{~nm}$ for each brain slice. We acquired three-dimensional 16-bit data stacks of $2048 \times 2048 \times 120$ pixels from five different positions in the frontal cortex at a lateral resolution of $0.17 \mu \mathrm{m} /$ pixel and an axial resolution of $0.4 \mu \mathrm{m} /$ pixel. To quantify Iba- 1 positive brain volume fraction, hereinafter referred as microglia brain fraction, as well as plaque density and size, we utilized custom-written Matlab software (MathWorks, Natick, USA). The detailed method was described previously [34].

Local background subtraction was used to diminish intensity variations between different stacks. Subsequently, microglia cells were identified by applying the 90th percentile as minimal-intensity threshold. Noise was excluded by applying a connected component analysis excluding patches of contiguous voxels smaller than $1 \mu^{3}$. Analyses were performed by an operator who was blind to the $\mu$ PET results.

\section{Statistics}

The associations between $\mu \mathrm{PET}$ readouts ( $\Delta \%, Z$-score, $Z$-score difference) and age were characterized by applying linear, logarithmic, and quadratic regression analyses as implemented in SPSS (SPSS Version 24, IBM SPSS Software, IBM, Armonk, New York). In cases with several statistically significant fits $(p<0.05)$, 
the best curve fitting model was determined by applying the Akaike Information Criterion (AIC) [35]. If the AIC proved indifferent between two models, we chose the one with the higher $R^{2}$ value. Statistics of histological analyses were calculated in Prism 7.01 (GraphPad Software, San Diego, CA, USA). Statistical comparison of the microglia fraction between different plaque radii was performed for the highest microglia occupancy in the vicinity to the plaque border [6]. Data was tested for normal distribution using the D'Agostino and Pearson omnibus test. Intergroup comparisons were performed using the two-tailed unpaired Student's $t$ test. For correlation of plaque size and microglia brain fraction, the variables were compared across groups using one-way analysis of variance (ANOVA). All specifications of $\mathrm{n}$ state the number of biological replicates. All results are presented as mean \pm standard error of mean (SEM).

\section{Results \\ Microglial response saturates relatively to ongoing amyloidosis during aging}

First, we analyzed serial changes of TSPO and fibrillar amyloidosis by dual tracer $\mu \mathrm{PET}$ to characterize the AD mouse model through molecular imaging. Both TSPO- $\mu$ PET $(+2.8 \pm 2.4 \%$ per month $)$ and A $\beta$-PET signals $(+2.9 \pm 2.5 \%$ per month direct comparison of TSPO-PET and A $\beta$-PET increase rates: $p=0.897$ ) increased strongly during aging of individual APP-SL70 mice. At late time points, the two markers were distinctly elevated when compared to wt mice (Fig. 2a-c) and there was a strong direct association between SUVR values of both PET tracers (quadratic fit, $R=$ $0.90, p<0.001)$. However, the percentage change of PET SUVR between baseline and +6.3 months as a function of starting age in APP-SL70 mice showed an inverted U-shape for TSPO binding (quadratic fit, $R=$ $0.69, p=0.014$, Fig. $2 \mathrm{~d}$ ) but a linear positive association for amyloidosis (linear fit, $R=0.50, p=0.048$, Fig. 2e). Thus, increases of microglial activity in aged APP-SL70 mice tended to reach a plateau whereas amyloidosis continued to progress even at late follow-up. Given the differences by tracer in $\mu$ PET alterations as functions of age, we aimed to compare directly the longitudinal time courses of TSPO activity and amyloidosis. To this end, we calculated standardized $Z$-scores for individual mice and for both tracers based on findings in age-matched wt controls. By this approach, we found the expected strong increases with age for TSPO activity (quadratic fit, $R=0.68, p<0.001$; Fig. $3 \mathrm{a}$ ) and fibrillar amyloidosis (quadratic fit, $R=0.86, p<0.001$, Fig. $3 \mathrm{~b})$. Next, $Z$-score differences (TSPO-A $\beta$ ) for all serial imaging time points were introduced as a measure of microglial activity relative to fibrillar amyloidosis.
Importantly, we observed a decreasing $Z$-score difference as a function of age in APP-SL70 mice (quadratic fit, $R=$ $0.66, p<0.001$, Fig. 3c), which clearly revealed that the microglial response to ongoing amyloid deposition is relatively attenuated at the later ages $((Z$-score difference $<0)$ $\geq 12.2$ months of age) of the mouse model. Notably, this effect was not only observed at the group level but was also distinguishable in single animals (Fig. 3d).

\section{Plaque-associated microglial brain fraction decreases with increasing plaque size during aging}

To confirm and extend observed in vivo results, methoxy-X04 staining of fibrillar $A \beta$ as well as Iba- 1 staining of microglia were performed in mid- (11.4 to 12.7 months) and late-aged (13.6 to 15.3 months) APP-SL70 mice after the final $\mu$ PET-scans. In line with previous studies, our immunohistochemical analysis showed that the observed increase in the $A \beta-\mu$ PET signal is indicative of plaque growth rather than increased plaque density (Fig. 4a, b). In late-aged APP-SL70, mice the mean plaque radius was significantly elevated by approximately $3 \mu \mathrm{m}$ compared to that in mid-aged mice $\left(t_{(14)}=5.86, p<0.0001\right.$, two-tailed Students $t$ test, Fig. $4 \mathrm{~b})$, whereas plaque density in both age groups remained unchanged at approximately $3600 / \mu \mathrm{m}^{3}\left(t_{(14)}\right.$ $=0.33, p=0.746$, two-tailed Students $t$ test, Fig. $4 \mathrm{c}$ ). Furthermore, plaque size distribution analysis showed a shift towards larger radii in late-aged compared to mid-aged transgenic mice (Fig. 4a).

Microglia proliferation in plaque-free areas and their migration towards $A \beta$ plaques has already been shown to occur in AD model mice [12], which results in an increased number of microglial cells surrounding amyloid deposits [36]. However, our standardized $\mu$ PET analysis revealed a decrease of the TSPO signal in direct relation to the increasing amyloid signal with aging (Fig. 3c). To assess the molecular relationship between plaque growth and microglial brain fraction, we applied custom-written MATLAB cluster analysis for automated morphological detection by applying the 90th percentile as the minimal intensity threshold for identifying microglial cells. We analyzed a total of 1312 plaques ranging in radius from $3 \mu \mathrm{m}$ to $30 \mu \mathrm{m}$ and identified their associated microglial cells via immunofluorescence. Representative plaques of several sizes are illustrated in Fig. 5. Interestingly, when we calculated the mean volume occupied by Iba1-positive microglia in consecutive $1-\mu \mathrm{m}$-thick layers around the plaque border up to $20 \mu \mathrm{m}$ distance, we observed a maximum of microglial brain fraction near small plaques (radii between 3 and $4 \mu \mathrm{m}$ ), while the microglia brain fraction decreased with increasing plaque radius $(F(5,16)=11.87, p<0.0001$, one-way ANOVA, (Fig. $4 \mathrm{~d}$, e, Fig. 5). We conclude that the observed age-related 

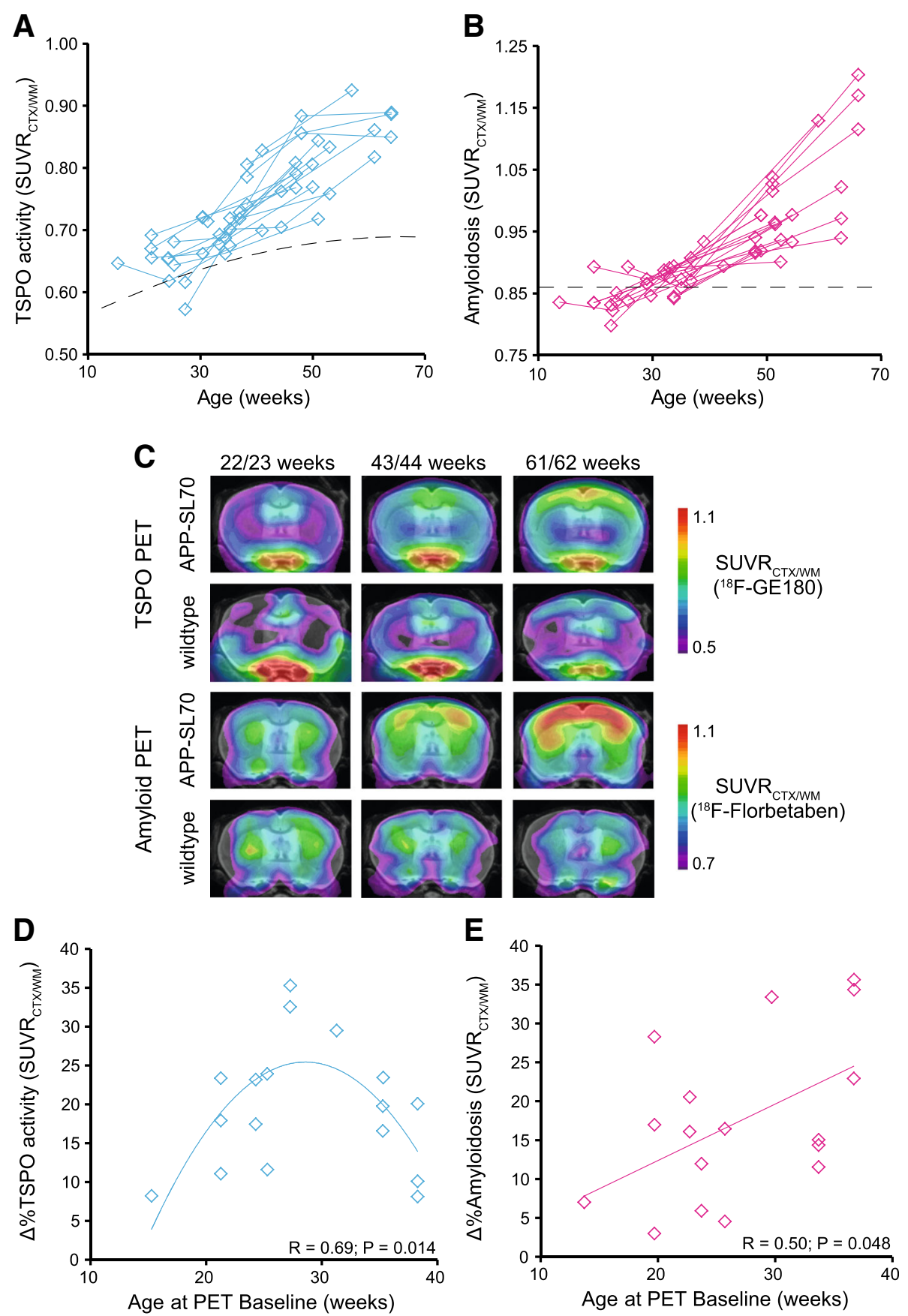

Fig. 2 Microglial response increased but saturated relative to ongoing amyloidosis during aging. Plots show cortical Standardized Uptake Value Ratio (SUVR) of $\left[{ }^{18} \mathrm{~F}\right]$-GE180 (TSPO-activity) (a) and $\left[{ }^{18} \mathrm{~F}\right.$-florbetaben (amyloidosis) (b) in APP-SL70 mice at different ages (B) APP-SL70 indicate increasing cortical amyloidosis and $18 \mathrm{kDa}$ translocator protein (TSPO) binding during aging (c). Percentage change for both PET tracers between baseline and + 6.3 months as a function of baseline age in APP-SL70 mice and wt reveal an inverted U-shape for TSPO activity (quadratic fit, $R=0.69, p=0.014$ (d) but a linear positive association for amyloidosis in APP-SL70 (linear fit, $R=0.50, p=0.048$ (e). N=17

decrease of the TSPO- $\mu$ PET signal relative to the $\mathrm{A} \beta-\mu \mathrm{PET}$ signal was likely driven by a decrease in the microglial brain fraction around large plaques, which came to predominate in late-aged APP-SL70 mice (Fig. 4b).
Microglial brain fraction in the plaque-free cortical brain parenchyma of APP-SL70 mice was less compared to wt mice

It is well acknowledged that microglial cells are essential for proper brain function and that microglia can 

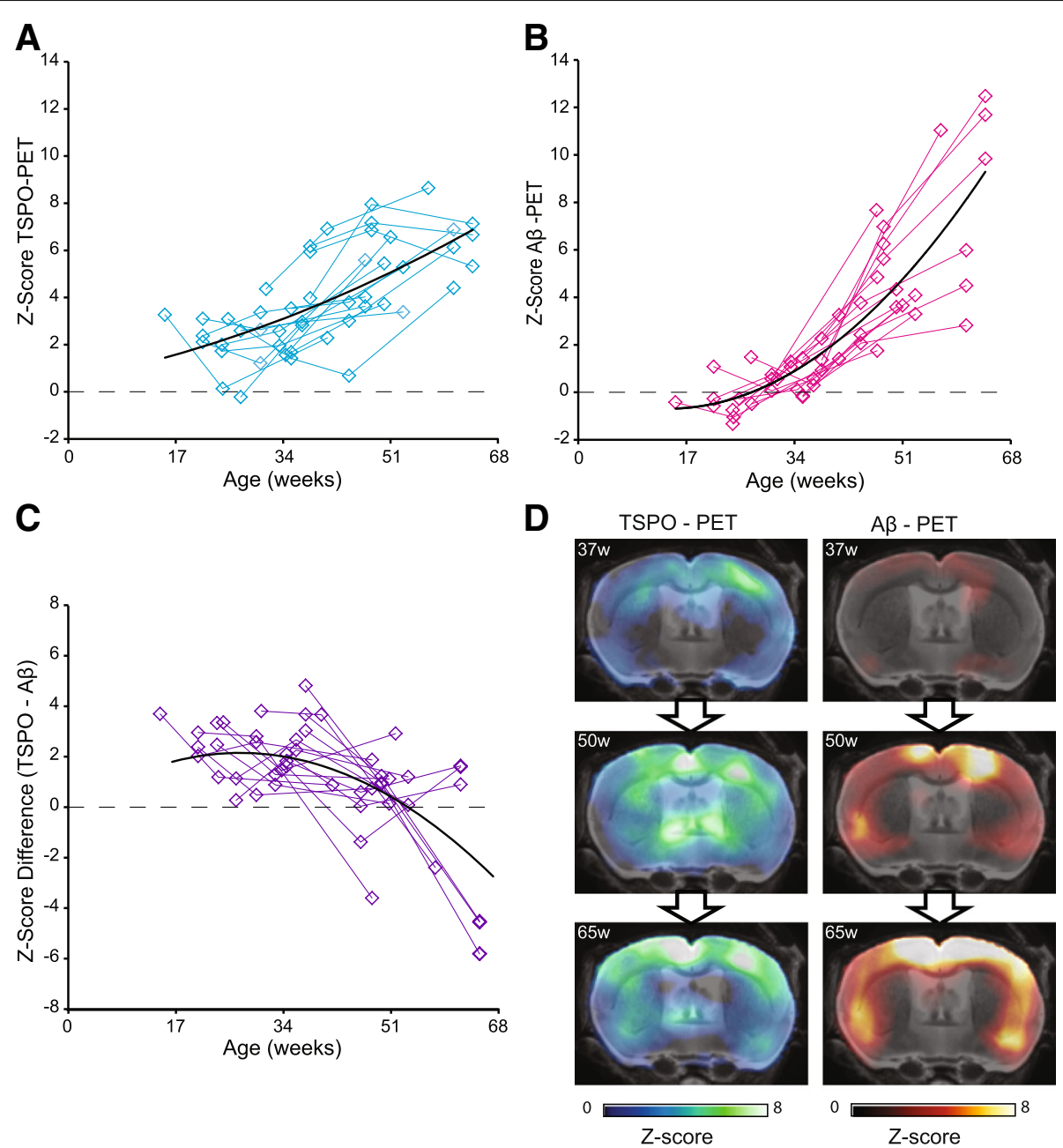

Fig. 3 Standardized $\mu$ PET analysis (Z-scores) TSPO activity and amyloidosis. Standardized TSPO activity (a; quadratic fit, $R=0.68, p<0.001)$ and standardized fibrillar amyloidosis ( $\mathbf{b}$; quadratic fit, $R=0.86, p<0.001$ ) indicate an increase with aging. The direct comparison of both standardized tracer signals reveals a decrease in the $Z$-score differences (TSPO-A $\beta$ ) with aging (c; quadratic fit, $R=0.66, p<0.001$ ). $\mathbf{d}$ Exemplary findings of a single APP-SL70 mouse show that microglia response is overwhelmed by ongoing amyloid deposition. $N=16$

rapidly proliferate in response to a wide range of central nervous system insults [37, 38]. In this context, a recent study of microglial turnover and proliferation in AD showed comparable rates of proliferation and loss for plaque-associated microglial cells indicating a steady-state, while non-plaque-associated microglial cells showed a threefold higher proliferation rate. In contrast, wt mice show only moderate rates of microglial cell proliferation and loss [12]. In the present study, we observed an increase in microglial brain fraction in APP-SL70 mice only in proximity to the plaque border, starting at a maximal distance of $20 \mu \mathrm{m}$ (Fig. 6a). Surprisingly, we detected a significantly lower microglial brain fraction distant to plaques in the APP-SL70 mice compared to the microglial brain fraction in wt mice $\left(t_{(25)}=2.18, p<0.05\right.$, two-tailed Students $t$ test, Fig. 6b). It is well-known that microglial cells are activated by $\mathrm{A} \beta$ deposits and actively migrate towards the plaque within 1 to 2 days after the initial formation of an amyloid deposit [6, 39]. Regarding the threefold higher proliferation rate of non-plaque-associated microglial cells, the net microglial loss distal to plaques in APP-SL70 compared to wt mice, while surprising, is conspicuous in our double-labelling studies (Fig. 6c).

\section{Discussion}

We present the first longitudinal in vivo dual tracer $\mu$ PET study aiming to directly compare the time courses of microglial activation and fibrillar amyloidosis with age in a transgenic amyloid mouse model. Our results clearly indicate that both biomarkers increase with age, but that microglial activation is disproportionately elevated at an early age and seems to 

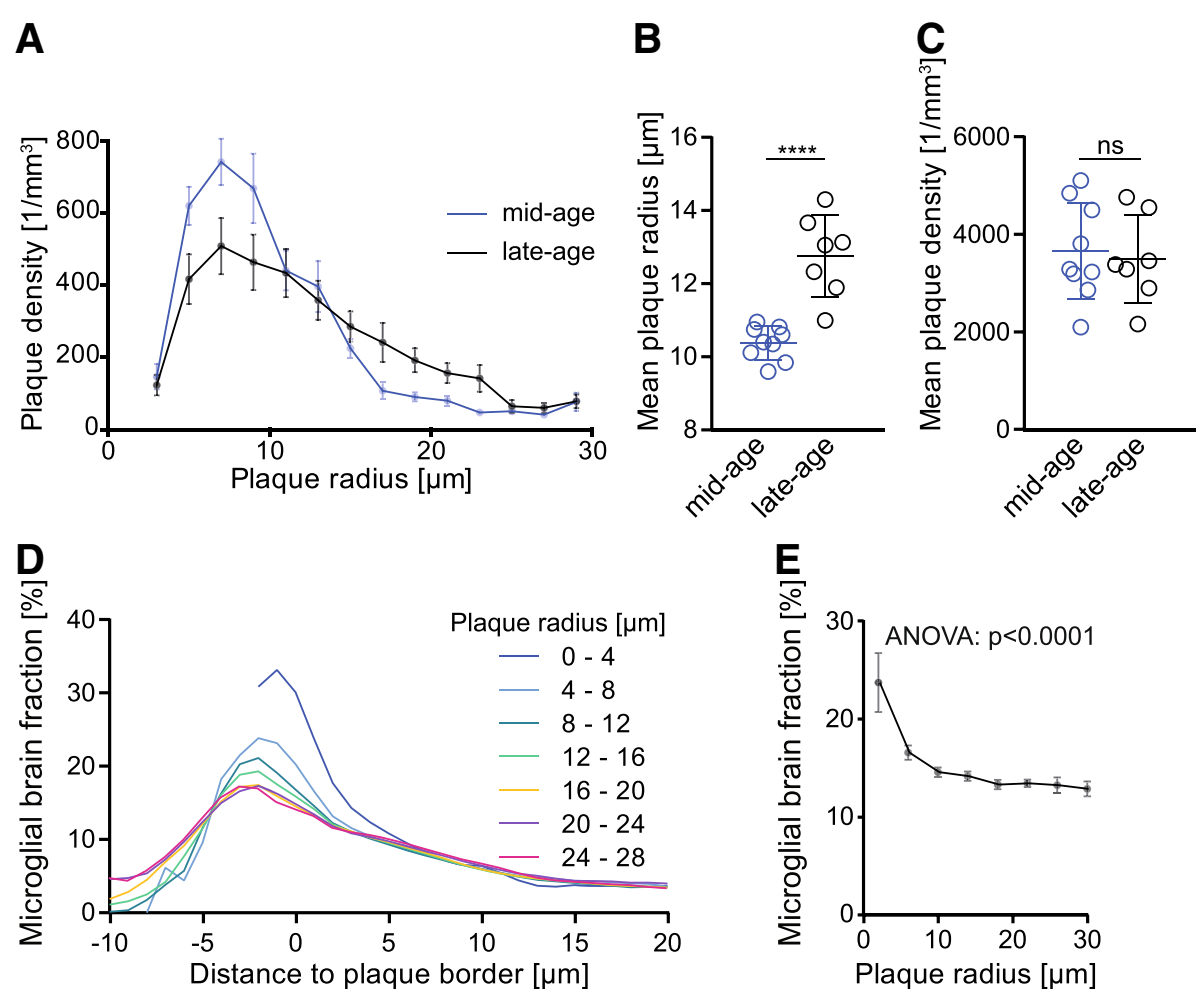

Fig. 4 Molecular elucidation of in vivo $\mu$ PET findings by terminal immunohistochemistry. a Frequency distribution of plaque radii in mid-aged (11.4 to 12.7 months) and late-aged (13.6 to 15.3 months) APP-SL70 mice. The mean plaque radius (b) is significantly higher in the late-aged cohort when compared to mid-aged APP-SL70 mice ( $p<0.0001$, two-tailed Student's $t$ test), whereas the plaque density (c) did not indicate changes during aging $>12$ months in APP-SL70 mice ( $p=0.746$, two-tailed Student's $t$ test). $\mathbf{d}$ Correlation of microglial brain fraction with distance to plaque border and plaque size. Each profile represents the change of microglial brain fraction with distance to the border of plaques with defined radius. e Microglial brain fraction in the vicinity to the plaque border (radius $1 \mu \mathrm{m}$ ) decreased significantly with increasing plaque radius (one-way ANOVA, $F(5,16)=11.87, p<0.0001$ ). Data presented as mean \pm SEM; $n=7-9$ mice

saturate relative to amyloidosis, which continues to progress. Detailed immunohistochemical analyses revealed a significant decrease of microglial brain fraction around amyloid plaques with increasing plaque radius to be the cellular correlate of our in vivo $\mu \mathrm{PET}$ findings. Moreover, we found that the microglia brain fraction in the plaque-free brain parenchyma of APP-SL70 mice was lower than in wt mice. This depletion of microglial cells distal to plaques is likely related to the massive microglial migration towards zones of fibrillar $\mathrm{A} \beta$ deposition $[6,39]$.

With this serial in vivo study, we aimed to investigate longitudinal relationships between microglial activation and amyloidosis during the life course of the APP-SL70 AD mouse model. We performed dual-tracer small animal $\mu$ PET examinations with the novel tracer $\left[{ }^{18} \mathrm{~F}\right]$-GE180 for TSPO and $\left[{ }^{18} \mathrm{~F}\right]$-florbetaben for fibrillar amyloidosis, in conjunction with immunohistochemical analyses after the final imaging studies. To enable a reliable comparison of the relationship between the two $\mu$ PET readouts, we took pains to develop a standard procedure for quantification, entailing a biphasic calculation method: First, we calculated standardized tracer specific $Z$-scores of individual mice at different time points by considering mean and standard deviation values of age-matched wt mice. We next calculated differences between TSPO- and $\mathrm{A} \beta$ - $\mu \mathrm{PET} Z$ Z-scores as a measure of microglial activation relative to fibrillar amyloidosis. We deemed this calculation of a difference score to be more reliable than a ratio method, as values close to zero would potentially have distorted the results at the onset of fibrillar amyloidosis in young mice. The two radioligands have different sensitivities for their specific targets, resulting in distinct detection thresholds unequal magnitudes of signal alterations during the progression of the AD model. To address these issues, we used the standardized $Z$-score calculation as our main endpoint. In fact, our analysis showed positive $Z$-score differences at early ages of APP-SL70 mice, which suggest that microglial activation precedes fibrillar amyloidosis at the onset of amyloid pathology. However, even with standardized $Z$-scores, there remains some possibility that this effect may be related to a higher sensitivity of 


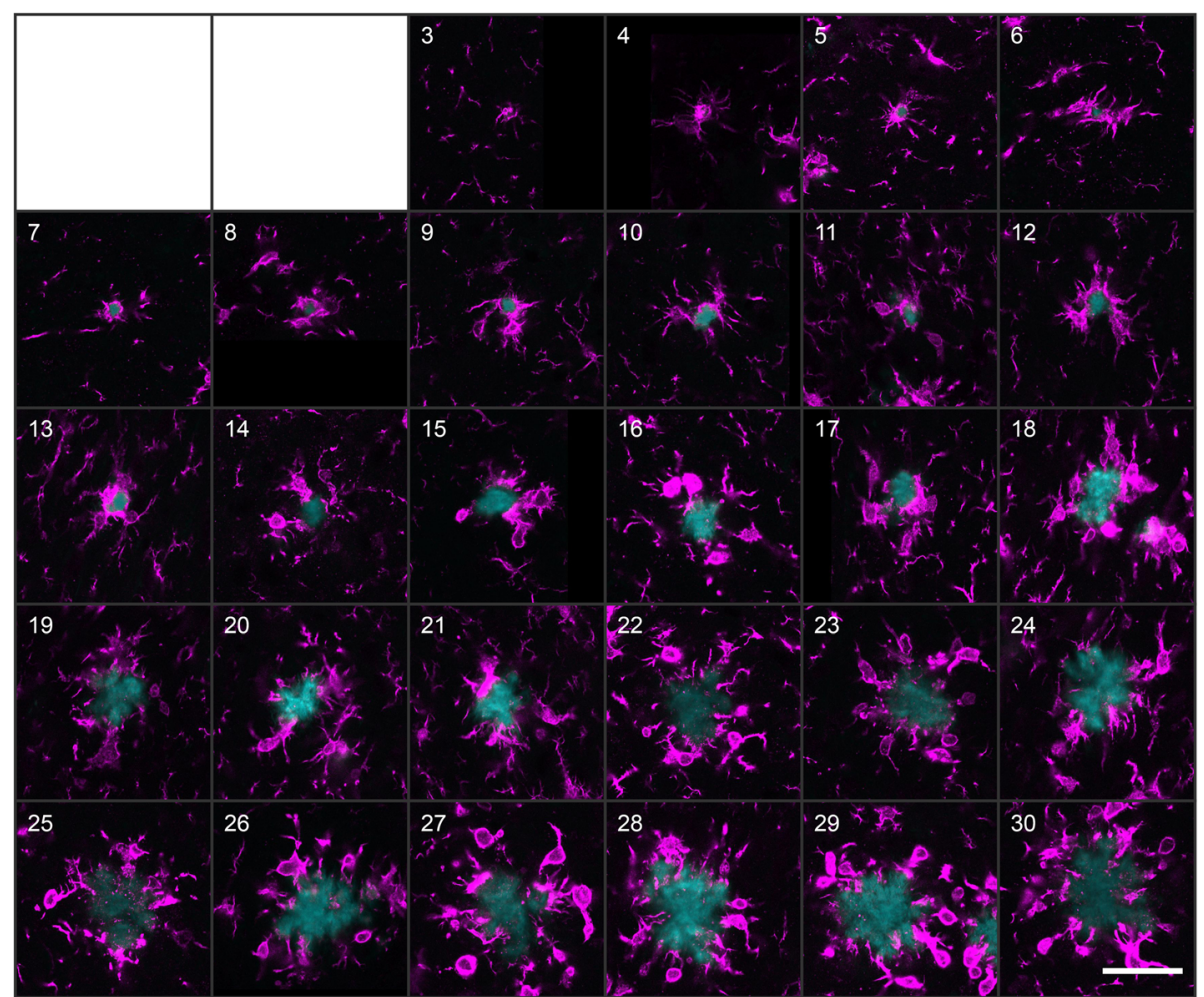

Fig. 5 Microglial brain fraction was maximal at small plaques. $\beta$-amyloid plaques of radius increasing from 3 to $30 \mu m$ are illustrated according to their size (white numeral in upper left corner). Each pane shows Methoxy-X04 stained plaque (cyan) together with the Iba-1 immunosignal (magenta). Scale bar $40 \mu \mathrm{m}$

the TSPO ligand to its target compared to the applied $\mathrm{A} \beta$ tracer. In contrast, due to its baseline dependency, the longitudinal decrease of the $Z$-score difference towards late ages is a rather compelling readout unlikely to be biased by possible tracer sensitivity differences. Furthermore, ceiling effects are unlikely as far higher magnitudes of TSPO activation and amyloidosis can be detected with these tracers in other circumstances [31, 40]. Thus, our serial dual tracer $\mu$ PET imaging proves that microglial activation saturates during an ongoing fibrillar amyloid deposition in this mouse model (Figs. 2 and 3). Our PET findings are absolutely in line with a recently observed plateau during TSPO PET imaging in aged APP23 mice by the same radioligand [41]. The results are also in line with findings for other biomarkers of microglial function, i.e., the peak in sTrem2 levels in cerebrospinal fluid of patients with mild cognitive impairment [42], followed by a drop in patients who have converted to dementia [43]. Even more importantly, computed longitudinal courses of sTrem2 in individuals with dominantly inherited AD decrease after symptom onset whereas amyloid deposition continues to progress [44], thus concurring with the presently observed relations between TSPO expression and fibrillar amyloidosis in aging APP-SL70 mice.
Although an important strength of $\mu$ PET lies in its fitness for longitudinal monitoring and target quantification, molecular imaging has limitations in spatial resolution and in its applicability for resolving mechanistic processes. For this reason, we supplemented $\mu$ PET with a detailed immunohistochemical study of activated microglia and histological staining of fibrillar $\mathrm{A} \beta$, which together supported automatized volumetric computations. Our data clearly indicate that microglia fraction adjacent to plaques declines with increasing plaque size (Fig. 4d). Given that plaque size but not density increases with advanced age (Fig. 4b, c), it seems obvious that the microglial activity must decrease relative to fibrillar amyloidosis over time. We validated these findings by comparing mid- and late aged APP-SL70 groups, concluding that the decreasing microglial brain fraction with increasing plaque size is consistent with our $\mu$ PET results in vivo. Since a recent study showed that brain location of microglia is a relevant factor for its morphological classification [45], our specific analysis of frontal cortical microglia cells in wt and APP-SL70 mice seems appropriate as it matched to the regional PET analysis.

So far, it remains unclear when and why microglial activity decreases adjacent to plaques. It is known that 

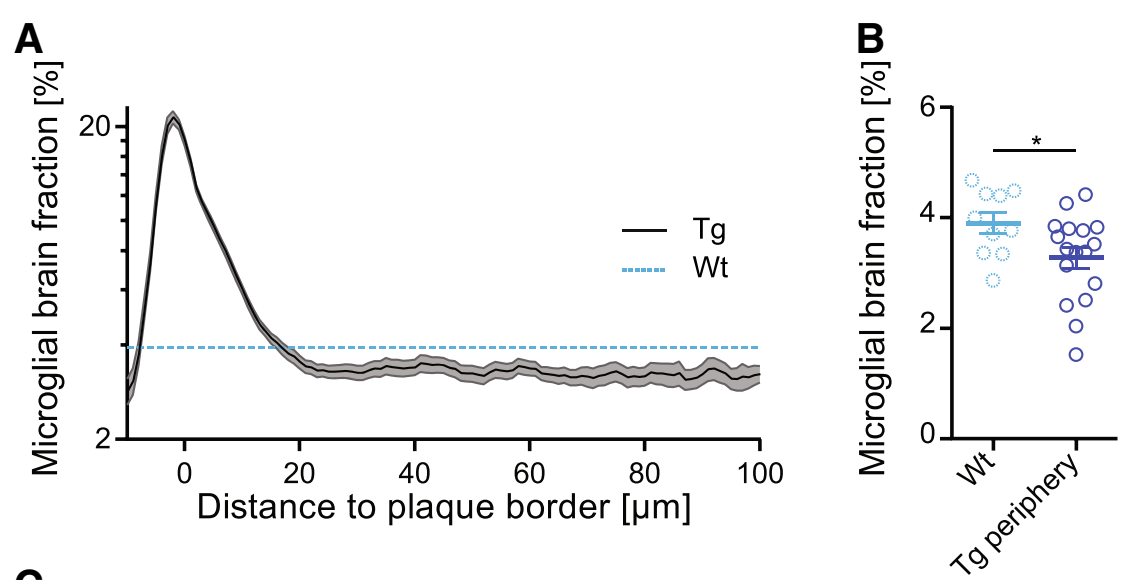

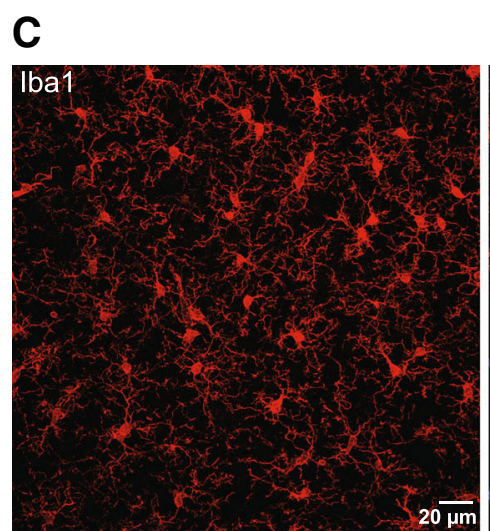

Wt

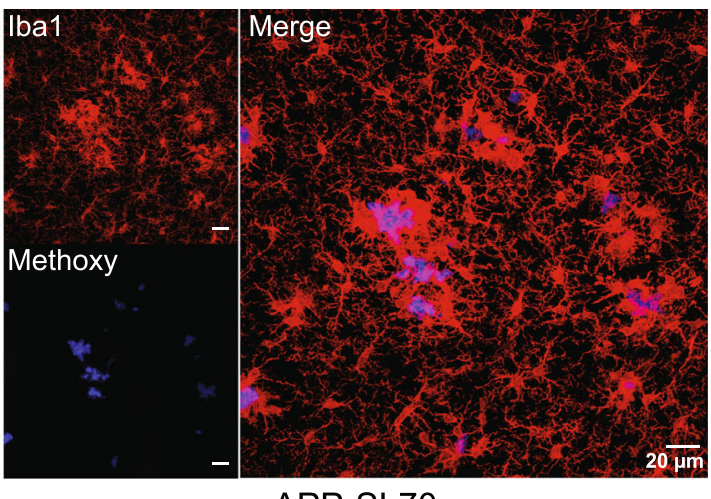

APP-SL70

Fig. 6 Microglial brain fraction decreased in the plaque-free cortical brain parenchyma of APP-SL70 mice. a Microglial brain fraction as a function of the distance to the plaque border in APP-SL70 mice (black line) when compared to the mean microglial brain fraction in wildtype (wt) mice (dotted blue line). $\mathbf{b}$ Direct comparison of microglial brain fraction of APP-SL70 mice (>30 $\mu \mathrm{m}$ from plaque borders) and wt mice (mean). Microglial brain fraction is significantly reduced compared to wt mice $(p<0.05$, two-tailed Student's $t$ test). $\mathbf{c}$ lba- 1 immunofluorescence staining in a wt mouse aged 16 months in comparison to a double staining of Iba-1 and methoxy-X04 stained plaque in APP-SL70 mouse aged 15 months. Data are presented as mean \pm SEM; $n=10-17$

microglia migrate within 1-2 days towards newly formed amyloid deposits, where they promote the clearance and phagocytosis of $A \beta$ by expressing certain cell-surface receptors [6-8]. However, during disease progression, plaque-associated microglial cells show a decrease in fiber mobility [46], lower expression of A $\beta$-binding receptors [11], and moreover also show a threefold higher mortality rate compared to nonplaque associated microglial cells [12]. Nonetheless, the same in vivo study reported a threefold higher proliferation rate of microglia distal to plaques in $\mathrm{AD}$ compared to wt mice, suggesting that new microglial cells migrate from the periphery to the plaque border [12]. However, we observed even lower microglial brain fraction distal to plaques of aged APP-SL70 mice compared to wt animals (Fig. 6). We conclude that the rate of microglia cell migration towards $A \beta$ depositions in APP-SL70 mice eventually exceeds the rate of proliferation of microglia cells staying in the peripheral zone. With aging, this potentially leads to an exhaustion of microglial cells for migration towards $A \beta$ depositions and, together with the increased rate of microglial cell loss around plaques [12], this may explain the observed decrease in microglia brain fraction with increasing plaque radius.

As a limitation of this study, we note that the amyloid tracer does not distinguish soluble and oligomeric $A \beta$. Thus, we cannot disentangle if there is a stronger association between microglia and soluble or oligomeric proportions of $A \beta$, which could show different growth rates with aging. Furthermore, given the nature of our longitudinal PET design, we were not able to acquire immunohistochemistry from mice at younger ages but instead focused on the late stage of the disease. Proliferation rates, mortality, and spatial distribution of microglia during their whole life cycle should therefore receive attention in future cross-sectional designs. 


\section{Conclusion}

Taken together, findings of this preclinical study in transgenic mice reveal the individual trajectories of microglial activation in relation to $A \beta$ deposition, thus providing important information about the staging of AD-like pathology, which could guide human clinical research. The translation of findings in animal models to human disease is challenging, but the strong bidirectional translational science potential of $\mu \mathrm{PET}$ findings to clinical PET holds great promise to dramatically advance our understanding of AD.

\begin{abstract}
Abbreviations
AD: Alzheimer's disease; AIC: Akaike Information Criterion; ANOVA: Analysis of variance; $A \beta$ : $\beta$-amyloid; GBq: Gigabecquerel; MBq: Megabecquerel; p.i: Post injection; PBR: Peripheral benzodiazepine receptor; SEM: Standard error of mean; SUVR: Standardized uptake value ratio; TSPO: 18 kDa translocator protein; VOI: Volume of interest; wt: Wildtype; $\Delta \%$ : Percentage change; HPET: Small animal positron emission tomography
\end{abstract}

\section{Acknowledgements}

We thank K. Bormann-Giglmaier and N. Lachner for their excellent technical support and animal care. Manuscript editing was provided by Inglewood Biomedical Editing. G.E. made GE-180 cassettes available through an early access model.

\section{Funding}

The study was financially supported by the SyNergy Cluster (J.H., P.B., C.H., and A.R.) and by the Deutsche Forschungsgemeinschaft (DFG) by a dedicated PET imaging grant to M.B. and A.R. (BR4580/1-1 \& RO5194/1-1).

\section{Availability of data and materials}

The datasets used and/or analyzed during the current study are available from the corresponding author on reasonable request.

\begin{abstract}
Authors' contributions
TB and CF performed the majority of PET and immunohistochemistry experiments. FP invented the Matlab driven $3 D$ analysis of plaque and microglia. MB, TB, CF, MD, and FP analyzed and quantified the data. NLA and PB performed the interpretation of the PET data. SL and FJG performed and improved radiochemistry. $\mathrm{KB}$ and $\mathrm{LO}$ contributed to the generation of the mouse model and helped to interpret the findings in the context of the model. MB, TB, and FP interpreted the data. $A R, J H$, and $M B$ contributed to the conception and design of the study. $\mathrm{TB}$ and $\mathrm{MB}$ wrote the manuscript. BU-S supervised the study as a veterinarian. All authors participated in the generation of the original data, added important intellectual content to the manuscript, and provided critical assessment of the current manuscript. All authors read and approved the final manuscript.
\end{abstract}

\section{Ethics approval and consent to participate}

All experiments were carried out in compliance with the National Guidelines for Animal Protection, Germany, with the approval of the regional Animal Care Committee of the Government of Oberbayern (Regierung Oberbayern) and were overseen by a veterinarian. Animal experiments were conducted in accordance with the guidelines EU Directive 2010/63/EU for animal experiments.

\section{Consent for publication}

Not applicable.

\section{Competing interests}

The authors Laurence Ozmen and Karlheinz Baumann are employed by company Roche, Basel, Switzerland. All other authors declare no financial or competing interests.

\section{Publisher's Note}

Springer Nature remains neutral with regard to jurisdictional claims in published maps and institutional affiliations.

\section{Author details}

'Department of Nuclear Medicine, University Hospital, LMU Munich, Marchioninistraße 15, 81377 Munich, Germany. ${ }^{2}$ German Center for Neurodegenerative Diseases (DZNE) Munich, Feodor-Lynen-Str. 17, 81377 Munich, Germany. ${ }^{3}$ Roche, Pharma Research and Early Development, NORD DTA / Neuroscience Discovery, Roche Innovation Center Basel, F. Hoffmann-La Roche Ltd., Grenzacherstrasse 124, CH-4070 Basel, Switzerland. ${ }^{4}$ Department of Nuclear Medicine, Inselspital, University Hospital Bern, Freiburgstrasse 4, 3010 Bern, Switzerland. ${ }^{5}$ Center of Neuropathology and Prion Research, Feodor-Lynen-Straße 23, 81377 Munich, Germany. ${ }^{6}$ Munich Cluster for Systems Neurology (SyNergy), Munich, Germany.

Received: 23 August 2018 Accepted: 26 October 2018 Published online: 06 November 2018

References

1. Perry VH, Gordon S. Macrophages and microglia in the nervous system. Trends Neurosci. 1988;11:273-7.

2. Dickson DW, Farlo J, Davies P, Crystal H, Fuld P, Yen SH. Alzheimer's disease. A double-labeling immunohistochemical study of senile plaques. Am J Pathol. 1988;132:86-101.

3. Selkoe DJ. The origins of Alzheimer disease: a is for amyloid. JAMA. 2000; 283:1615-7

4. Mott RT, Hulette CM. Neuropathology of Alzheimer's disease. Neuroimaging Clin N Am. 2005;15:755-65, ix. https://doi.org/10.1016/j.nic.2005.09.003.

5. Nimmerjahn A, Kirchhoff F, Helmchen F. Resting microglial cells are highly dynamic surveillants of brain parenchyma in vivo. Science. 2005;308:1314-8. https://doi.org/10.1126/science.1110647.

6. Bolmont T, Haiss F, Eicke D, Radde R, Mathis CA, Klunk WE, et al. Dynamics of the microglial/amyloid interaction indicate a role in plaque maintenance. J Neurosci. 2008;28:4283-92. https://doi.org/10.1523/ JNEUROSCI.4814-07.2008.

7. Yan SD, Chen X, Fu J, Chen M, Zhu H, Roher A, et al. RAGE and amyloidbeta peptide neurotoxicity in Alzheimer's disease. Nature. 1996;382:685-91. https://doi.org/10.1038/382685a0.

8. El Khoury J, Hickman SE, Thomas CA, Loike JD, Silverstein SC. Microglia, scavenger receptors, and the pathogenesis of Alzheimer's disease. Neurobiol Aging. 1998;19:\$81-4.

9. El Khoury J, Toft M, Hickman SE, Means TK, Terada K, Geula C, Luster AD. Ccr2 deficiency impairs microglial accumulation and accelerates progression of Alzheimer-like disease. Nat Med. 2007;13:432-8. https:// doi.org/10.1038/nm1555.

10. Lee CYD, Landreth GE. The role of microglia in amyloid clearance from the AD brain. J Neural Transm (Vienna). 2010;117:949-60. https://doi.org/10. 1007/s00702-010-0433-4

11. Hickman SE, Allison EK, El Khoury J. Microglial dysfunction and defective beta-amyloid clearance pathways in aging Alzheimer's disease mice. J Neurosci. 2008;28:8354-60. https://doi.org/10.1523/JNEUROSCI.0616-08.2008.

12. Füger $P$, Hefendehl JK, Veeraraghavalu $K$, Wendeln A-C, Schlosser $C$, Obermüller $U$, et al. Microglia turnover with aging and in an Alzheimer's model via long-term in vivo single-cell imaging. Nat Neurosci. 2017;20: 1371-6. https://doi.org/10.1038/nn.4631.

13. Matthews PM, Rabiner EA, Passchier J, Gunn RN. Positron emission tomography molecular imaging for drug development. Br J Clin Pharmacol. 2012:73:175-86. https://doi.org/10.1111/j.1365-2125.2011.04085.x.

14. Zimmer ER, Parent MJ, Cuello AC, Gauthier S, Rosa-Neto P. MicroPET imaging and transgenic models: a blueprint for Alzheimer's disease clinical research. Trends Neurosci. 2014;37:629-41. https://doi.org/10.1016/j.tins. 2014.07.002.

15. Rominger A, Brendel M, Burgold S, Keppler K, Baumann K, Xiong G, et al. Longitudinal assessment of cerebral $\beta$-amyloid deposition in mice overexpressing Swedish mutant $\beta$-amyloid precursor protein using 18Fflorbetaben PET. J Nucl Med. 2013;54:1127-34. https://doi.org/10.2967/ jnumed.112.114660.

16. Brendel M, Jaworska A, Herms J, Trambauer J, Rötzer C, Gildehaus F-J, et al. Amyloid-PET predicts inhibition of de novo plaque formation upon chronic -secretase modulator treatment. Mol Psychiatry. 2015;20:1179-87. https:// doi.org/10.1038/mp.2015.74.

17. Barthel H, Gertz H-J, Dresel S, Peters O, Bartenstein P, Buerger K, et al. Cerebral amyloid- $\beta$ PET with florbetaben (18F) in patients with Alzheimer's disease and healthy controls: a multicentre phase 2 diagnostic study. The 
Lancet Neurology. 2011;10:424-35. https://doi.org/10.1016/S14744422(11)70077-1.

18. Mirzaei N, Tang SP, Ashworth S, Coello C, Plisson C, Passchier J, et al. In vivo imaging of microglial activation by positron emission tomography with (11)CPBR28 in the 5XFAD model of Alzheimer's disease. Glia. 2016;64:9931006. https://doi.org/10.1002/glia.22978.

19. Cumming P, Burgher B, Patkar O, Breakspear M, Vasdev N, Thomas $P$, et al. Sifting through the surfeit of neuroinflammation tracers. J Cereb Blood Flow Metab. 2018;38:204-24. https://doi.org/10.1177/0271678X17748786.

20. Dickens AM, Vainio S, Marjamäki P, Johansson J, Lehtiniemi P, Rokka J, et al. Detection of microglial activation in an acute model of neuroinflammation using PET and radiotracers 11C-(R)-PK11195 and 18F-GE-180. J Nucl Med. 2014;55:466-72. https://doi.org/10.2967/jnumed.113.125625.

21. James ML, Belichenko NP, Nguyen T-W, Andrews LE, Ding Z, Liu H, et al. PET imaging of translocator protein $(18 \mathrm{kDa})$ in a mouse model of Alzheimer's disease using N-(2,5-dimethoxybenzyl)-2-18F-fluoro-N-(2phenoxyphenyl)acetamide. J Nucl Med. 2015;56:311-6. https://doi.org/10. 2967/jnumed.114.141648.

22. Liu B, Le KX, Park M-A, Wang S, Belanger AP, Dubey S, et al. In vivo detection of age- and disease-related increases in neuroinflammation by 18F-GE180 TSPO MicroPET imaging in wild-type and Alzheimer's transgenic mice. J Neurosci. 2015;35:15716-30. https://doi.org/10.1523/JNEUROSCI. 0996-15.2015.

23. Wickstrøm T, Clarke A, Gausemel I, Horn E, Jørgensen K, Khan I, et al. The development of an automated and GMP compliant FASTlab ${ }^{T M}$ synthesis of (18) FGE-180; a radiotracer for imaging translocator protein (TSPO). J Labelled Comp Radiopharm. 2014;57:42-8. https://doi.org/10.1002/jlcr.3112.

24. Chen M-K, Guilarte TR. Translocator protein 18 kDa (TSPO): molecular sensor of brain injury and repair. Pharmacol Ther. 2008;118:1-17. https://doi.org/10. 1016/.jpharmthera.2007.12.004

25. Cosenza-Nashat M, Zhao M-L, Suh H-S, Morgan J, Natividad R, Morgello S, Lee SC. Expression of the translocator protein of $18 \mathrm{kDa}$ by microglia, macrophages and astrocytes based on immunohistochemical localization in abnormal human brain. Neuropathol Appl Neurobiol. 2009;35:306-28. https://doi.org/10.1111/j.1365-2990.2008.01006.x.

26. Scarf $A M$, Ittner $L M$, Kassiou $M$. The translocator protein $(18 \mathrm{kDa})$ : central nervous system disease and drug design. J Med Chem. 2009;52:581-92. https://doi.org/10.1021/jm8011678.

27. Venneti S, Wagner AK, Wang G, Slagel SL, Chen X, Lopresti BJ, et al. The high affinity peripheral benzodiazepine receptor ligand DAA1106 binds specifically to microglia in a rat model of traumatic brain injury: implications for PET imaging. Exp Neurol. 2007;207:118-27. https://doi.org/10.1016/j. expneurol.2007.06.003.

28. Venneti S, Wiley CA, Kofler J. Imaging microglial activation during neuroinflammation and Alzheimer's disease. J Neurolmmune Pharmacol. 2009;4:227-43. https://doi.org/10.1007/s11481-008-9142-2.

29. Brendel M, Probst F, Jaworska A, Overhoff F, Korzhova V, Albert NL, et al. Glial activation and glucose metabolism in a transgenic amyloid mouse model: a triple-tracer PET study. J Nucl Med. 2016;57:954-60. https://doi.org/ 10.2967/jnumed.115.167858.

30. Blanchard V, Moussaoui S, Czech C, Touchet N, Bonici B, Planche M, et al. Time sequence of maturation of dystrophic neurites associated with $A \beta$ deposits in APP/PS1 transgenic mice. Exp Neurol. 2003;184:247-63. https:// doi.org/10.1016/50014-4886(03)00252-8.

31. Brendel M, Jaworska A, Grießinger E, Rötzer C, Burgold S, Gildehaus F-J, et al. Cross-sectional comparison of small animal 18F-florbetaben amyloid-PET between transgenic AD mouse models. PLoS One. 2015;10:e0116678. https://doi.org/10.1371/journal.pone.0116678.

32. Overhoff F, Brendel M, Jaworska A, Korzhova V, Delker A, Probst F, et al. Automated spatial brain normalization and hindbrain white matter reference tissue give improved (18)F-Florbetaben PET quantitation in Alzheimer's model mice. Front Neurosci. 2016;10:45. https://doi.org/10.3389/ fnins.2016.00045.

33. Rominger A, Mille E, Zhang S, Böning G, Förster S, Nowak S, et al. Validation of the octamouse for simultaneous 18F-fallypride small-animal PET recordings from 8 mice. J Nucl Med. 2010;51:1576-83. https://doi.org/10. 2967/jnumed.110.078451

34. Peters F, Salihoglu H, Rodrigues E, Herzog E, Blume T, Filser $S$, et al. BACE1 inhibition more effectively suppresses initiation than progression of $\beta$ amyloid pathology. Acta Neuropathol. 2018;135:695-710. https://doi.org/10. 1007/s00401-017-1804-9.
35. Akaike H. A new look at the statistical model identification. IEEE Trans Automat Contr. 1974;19:716-23. https://doi.org/10.1109/TAC.1974.1100705.

36. Radde R, Bolmont T, Kaeser SA, Coomaraswamy J, Lindau D, Stoltze L, et al. Abeta42-driven cerebral amyloidosis in transgenic mice reveals early and robust pathology. EMBO Rep. 2006;7:940-6. https://doi.org/10.1038/sj.embor. 7400784.

37. Gómez-Nicola D, Fransen NL, Suzzi S, Perry VH. Regulation of microglial proliferation during chronic neurodegeneration. J Neurosci. 2013;33:248193. https://doi.org/10.1523/JNEUROSCl.4440-12.2013.

38. Graeber MB, López-Redondo F, Ikoma E, Ishikawa M, Imai Y, Nakajima K, et al. The microglia/macrophage response in the neonatal rat facial nucleus following axotomy. Brain Res. 1998;813:241-53.

39. Meyer-Luehmann M, Coomaraswamy J, Bolmont T, Kaeser S, Schaefer C, Kilger $E$, et al. Exogenous induction of cerebral beta-amyloidogenesis is governed by agent and host. Science. 2006;313:1781-4. https://doi.org/10. 1126/science.1131864

40. Zwergal A, Günther L, Brendel M, Beck R, Lindner S, Xiong $G$, et al. In vivo imaging of glial activation after unilateral labyrinthectomy in the rat: a 18FGE180-PET study. Front Neurol. 2017;8:665. https://doi.org/10.3389/fneur. 2017.00665.

41. López-Picón FR, Snellman A, Eskola O, Helin S, Solin O, Haaparanta-Solin M, Rinne JO. Neuroinflammation appears early on PET imaging and then plateaus in a mouse model of Alzheimer disease. J Nucl Med. 2018;59:50915. https://doi.org/10.2967/jnumed.117.197608.

42. Ulrich JD, Ulland TK, Colonna M, Holtzman DM. Elucidating the role of TREM2 in Alzheimer's disease. Neuron. 2017;94:237-48. https://doi.org/10. 1016/j.neuron.2017.02.042.

43. Suárez-Calvet M, Kleinberger G, Araque Caballero MÁ, Brendel M, Rominger A, Alcolea D, et al. sTREM2 cerebrospinal fluid levels are a potential biomarker for microglia activity in early-stage Alzheimer's disease and associate with neuronal injury markers. EMBO Mol Med. 2016;8:466-76. https://doi.org/10.15252/emmm.201506123.

44. Suárez-Calvet M, Araque Caballero MÁ, Kleinberger G, Bateman RJ, Fagan AM, Morris JC, et al. Early changes in CSF sTREM2 in dominantly inherited Alzheimer's disease occur after amyloid deposition and neuronal injury. Sci Transl Med. 2016;8:369ra178. https://doi.org/10.1126/scitransImed.aag1767.

45. Fernández-Arjona MDM, Grondona JM, Granados-Durán P, FernándezLlebrez P, López-Ávalos MD. Microglia morphological categorization in a rat model of neuroinflammation by hierarchical cluster and principal components analysis. Front Cell Neurosci. 2017;11:235. https://doi.org/10. 3389/fncel.2017.00235.

46. Krabbe G, Halle A, Matyash V, Rinnenthal JL, Eom GD, Bernhardt U, et al. Functional impairment of microglia coincides with beta-amyloid deposition in mice with Alzheimer-like pathology. PLoS One. 2013;8:e60921. https://doi. org/10.1371/journal.pone.0060921.
Ready to submit your research? Choose BMC and benefit from:
- fast, convenient online submission
- thorough peer review by experienced researchers in your field
- rapid publication on acceptance
- support for research data, including large and complex data types
- gold Open Access which fosters wider collaboration and increased citations
- maximum visibility for your research: over $100 \mathrm{M}$ website views per year
At BMC, research is always in progress.
Learn more biomedcentral.com/submissions 